\title{
FEM/FMBEM coupling for acoustic structure interaction and acoustic design sensitivity analysis with sound-absorbing materials
}

\author{
Y. M. Xu, H. B. Chen \& L. L. Chen \\ Department of Modern Mechanics, \\ University of Science and Technology of China, PR China
}

\begin{abstract}
For thin structures immersed in water, a full interaction between the structural domain and the fluid domain needs to be taken into account. In this work, the finite element method (FEM) is used to model the structure parts, while the boundary element method (BEM) is applied to the exterior acoustic domain. A coupling algorithm based on FEM and the wideband fast multipole BEM (FEM/Wideband FMBEM) is used for the simulation of acoustic-structure interaction. The Burton-Miller formulation is used to overcome the fictitious frequency problem when using a single Helmholtz boundary integral equation for exterior boundary-value problems. Structural-acoustic design sensitivity analysis is performed based on the coupling formulation. The design variable can be chosen as the material parameters, structure and fluid parameters, such as the fluid density, structural density, Poisson's ratio, Young's modulus, structural shape size and so on. Furthermore, the impact of sound-absorbing material on the scattering problem for structures underwater is researched. The acoustic admittance of the soundabsorbing material has also been chosen as the design variable for the sensitivity analysis. Numerical example is presented to demonstrate the validity and efficiency of the proposed algorithm.

Keywords: finite element method, fast multipole boundary element method, fluidstructure interaction, design sensitivity analysis, sound-absorbing material.
\end{abstract}




\section{Introduction}

Analysis of the acoustic radiation or scattering from elastic structure in fluid is a classical problem of underwater acoustics. Analytical solutions to the acoustic fluid-structure interaction problems are only available when the structure has simple geometry with simple boundary conditions [1]. For more complicated problems, it is impossible to find analytical solutions, and thus necessary to create efficient numerical methods. A suitable approach for the analysis of fluid-structure interaction problems is the coupled FEM/BEM $[2,3]$. But the coupling analysis based on FEM/Conventional BEM (CBEM) algorithm still represents the bottleneck of large computation cost, because the CBEM produces a dense and non-symmetrical coefficient matrix which induces $\mathrm{O}(\mathrm{N} 3)$ arithmetic operations to solve the system of equations directly, such as by using the Gauss elimination method. The fast multipole method (FMM) [4-8] has been presented to accelerate the solution of the CBEM system of equations and to decrease the memory requirement. The wideband FMM [9-11] could work accurately and efficiently at all frequencies. On the other hand, the implementation of a single Helmholtz boundary integral equation may have the difficulty of non-uniqueness for exterior boundary-value problems. The Burton-Miller method [12] could effectively overcome the non-unique solution problem. In this paper, the coupling algorithm FEM/Wideband FMBEM [13] is applied to solve the structural-acoustic interaction problems.

The sound-absorbing material has been widely used to decrease noise radiation. Special effort is devoted to numerically obtain the absorption coefficient and acoustic conductance. For the generalization of the boundary conditions [14], the acoustic admittance $\beta$ could be introduced, which tends to be zero at perfectly reflecting boundaries like a rigid wall [15]. On the other hand, for non-reflecting boundary conditions, $\beta=1$. The admittance could also be replaced by impedance which is defined as it's reciprocal. In this work, the acoustic admittance $\beta$ is introduced to extend the coupling algorithm FEM/Wideband FMBEM for the structural-acoustic interaction analysis with sound-absorbing material.

Passive noise control by modification of structure geometry moves more and more into the field of vision for designers. This structural-acoustic optimization shows high potential in minimization of radiated noise especially for thin shell geometries [16]. Acoustic design sensitivity analysis can provide information on how the geometry change affects the performance of the structure, so it is an important step. The coupling algorithm FEM/Wideband FMBEM has been applied to structural-acoustic sensitivity analysis based on the direct differentiation method [13]. In this paper, FEM/Wideband FMBEM is applied to the structuralacoustic sensitivity analysis with sound-absorbing material based on the finite difference method. The design variable could be set as the fluid density, structural density and so on. The formulation of sound pressure sensitivity for fluid-structure interaction is derived. A numerical example is presented to demonstrate the validity and efficiency of the proposed algorithm. 


\section{Structural-acoustic formulations}

\subsection{FEM formulations}

Assuming that a harmonic load with the excitation frequency $\omega$ is applied to the structure, the steady-state response can be calculated from the frequency-response analysis. The linear system of equations for the computation of the nodal displacement $\boldsymbol{u}$ is derived by:

$$
\left(\boldsymbol{K}+i \omega \boldsymbol{C}-\omega^{2} \boldsymbol{M}\right) \boldsymbol{u}=\boldsymbol{A} \boldsymbol{u}=\boldsymbol{f},
$$

in which $i=\sqrt{-1}, \boldsymbol{K}$ is the stiffness matrix, $\boldsymbol{C}$ is the damping matrix, $\boldsymbol{M}$ is the mass matrix, and $\boldsymbol{u}$ is the nodal displacement vector. Taking into account the acoustic pressure on the structure surface, an acoustic load $\boldsymbol{f}_{p}$ is applied along with the structural load $\boldsymbol{f}_{s}$, and the excitation can be expressed as:

$$
\boldsymbol{f}=\boldsymbol{f}_{s}+\boldsymbol{f}_{p}=\boldsymbol{f}_{s}+\boldsymbol{C}_{s f} \boldsymbol{p},
$$

and the coupling matrix $\boldsymbol{C}_{s f}$ can be expressed as:

$$
\boldsymbol{C}_{s f}=\int_{S_{\text {int }}} \boldsymbol{N}_{s}^{T} \boldsymbol{n} \boldsymbol{N}_{f} d S_{\text {int }},
$$

in which $\boldsymbol{N}_{s}$ and $\boldsymbol{N}_{f}$ denote the global interpolation functions for the structure and fluid domains, respectively, $\boldsymbol{n}$ is the surface normal vector, and $S_{i n t}$ is the interaction surface. By substituting equation (2) into equation (1), we can obtain the following formulation:

$$
\boldsymbol{u}=\boldsymbol{A}^{-1} \boldsymbol{f}_{s}+\boldsymbol{A}^{-1} \boldsymbol{C}_{s f} \boldsymbol{p}
$$

\subsection{BEM formulations}

Consider the Helmholtz equation governing a time-harmonic acoustic wave field:

$$
\nabla^{2} p(x)+k^{2} p(x)=0, \quad \forall x \in \Omega,
$$

in which $p$ is the acoustic pressure, $k$ is the wave number and $k=\omega / c, \omega$ is the angular frequency, and $c$ is the wave speed in the acoustic medium $\Omega$.

Reformulating the Helmholtz equation, we can get a boundary integral equation which is defined on the structure boundary $S$ :

$$
\int_{S} G(x, y) q(y) d S(y)-\int_{S} F(x, y) p(y) d S(y)=c(x) p(x)-p_{i}(x),
$$

in which $x$ is the source point, $y$ is the field point, the coefficient $c(x)$ is equal to $1 / 2$ when the boundary $S$ is smooth around the point $x, p_{i}(x)$ is the incident wave, $G(x, y)$ is the Green's function, $q(y)$ and $F(x, y)$ are the normal derivatives of $p(y)$ and $G(x, y)$, respectively. As for three dimensional acoustic wave problems, $G(x, y)$ and $F(x, y)$ are given as: 


$$
\begin{gathered}
G(x, y)=\frac{e^{i k r}}{4 \pi r}, \\
F(x, y)=\frac{e^{i k r}}{4 \pi r^{2}}(i k r-1) \frac{\partial r}{\partial n(x)},
\end{gathered}
$$

in which $r$ is the distance between the source point $x$ and the field point $y$. Dividing the boundary $S$ into $N$ elements, collecting the equations for all the collocation points located at the center of each element and expressing them in matrix form, we can get the linear algebraic equations, as follows:

$$
\boldsymbol{H p}=\boldsymbol{G} \boldsymbol{q}+\boldsymbol{p}_{i} .
$$

For Burton-Miller method, one can get the same form of last discretized formulation [13].

\subsection{FEM/BEM coupling formulations}

Assuming that the structure surface is coated with sound-absorbing material which is too thin to affect the structural stiffness matrix or mass matrix, $\boldsymbol{q}$ in equation (9) could be expressed as:

$$
\boldsymbol{q}=\boldsymbol{q}_{1}+\boldsymbol{q}_{2} .
$$

After applying the impedance boundary condition and the continuity boundary condition across the interaction surface respectively, $\boldsymbol{q}_{1}$ and $\boldsymbol{q}_{2}$ in equation (10) could be given as:

$$
\begin{gathered}
\boldsymbol{q}_{1}=\frac{\partial \boldsymbol{p}}{\partial \boldsymbol{n}}=i k \beta \boldsymbol{p}, \\
\boldsymbol{q}_{2}=-i \omega \rho_{f} \boldsymbol{v}_{s}=\omega^{2} \rho_{f} \boldsymbol{S}^{-1} \boldsymbol{C}_{f s} \boldsymbol{u},
\end{gathered}
$$

in which $\beta$ is the acoustic admittance, $\boldsymbol{S}=\int_{S_{\text {int }}} \boldsymbol{N}_{f}^{T} \boldsymbol{N}_{f} d S_{\text {int }}$ and $\boldsymbol{C}_{f s}=\boldsymbol{C}_{s f}^{T}$.

By combining equations (10)-(12) and substituting the combined equation into equations (9), we can get the following formulation:

$$
\boldsymbol{H p}=\boldsymbol{G}\left(i k \beta \boldsymbol{p}+\omega^{2} \rho_{f} \boldsymbol{S}^{-1} \boldsymbol{C}_{f s} \boldsymbol{u}\right)+\boldsymbol{p}_{i} .
$$

Combining equation (1) and equation (13), the coupled system of equations could be expressed as:

$$
\left[\begin{array}{cc}
\boldsymbol{A} & -\boldsymbol{C}_{s f} \\
-\omega^{2} \rho_{f} \boldsymbol{G} \boldsymbol{S}^{-1} \boldsymbol{C}_{f s} & \boldsymbol{H}-i k \beta \boldsymbol{G}
\end{array}\right]\left\{\begin{array}{l}
\boldsymbol{u} \\
\boldsymbol{p}
\end{array}\right\}=\left\{\begin{array}{l}
\boldsymbol{f}_{s} \\
\boldsymbol{p}_{i}
\end{array}\right\} .
$$

Direct iterations of equation (14) converge very slowly, and it takes considerable time and storage requirement to solve the system equations directly. It's also very difficult to obtain the numerical solutions with high accuracy. Instead of solving the non-symmetric linear equations by using an iterative solver, we 
propose the next approach. By substituting equation (4) into equation (13), we can obtain the coupled boundary element equation, as follows:

$$
(\boldsymbol{H}-i k \beta \boldsymbol{G}) \boldsymbol{p}-\boldsymbol{G} \boldsymbol{W} \boldsymbol{C}_{s f} \boldsymbol{p}=\boldsymbol{p}_{i}+\boldsymbol{G} \boldsymbol{W} \boldsymbol{f}_{s},
$$

in which $\boldsymbol{W}=\omega^{2} \rho_{f} \boldsymbol{S}^{-1} \boldsymbol{C}_{f s} \boldsymbol{A}^{-1}$. Directly solving the term $\boldsymbol{A}^{-1} \boldsymbol{C}_{s f} \boldsymbol{p}$ in equation (15) could consume considerable time. In this paper, we use the fast multipole method and the iterative solver generalized minimal residual method (GMRES) to accelerate the calculation of the solution to the coupled boundary element system equation. Assuming the current iterative solution to be $\boldsymbol{p}_{k}$, by calculating $\boldsymbol{C}_{s f} \boldsymbol{p}_{k}$, we can get a new vector $\boldsymbol{b}=\boldsymbol{C}_{s f} \boldsymbol{p}_{k}$. Then, the solution $\boldsymbol{A}^{-1} \boldsymbol{b}$ could be efficiently obtained when using a sparse direct solver to solve the linear equation $\boldsymbol{A x}=\boldsymbol{b}$, in which $\boldsymbol{A}^{-1} \boldsymbol{b}=\boldsymbol{x}$. The term $\boldsymbol{A}^{-1} \boldsymbol{f}_{s}$ in equation (15) could be calculated similarly.

\section{Acoustic design sensitivity analysis}

Equation (14) could be expressed as:

$$
\left[\begin{array}{cc}
\boldsymbol{A} & -\boldsymbol{C}_{s f} \\
-\omega^{2} \rho_{f} \boldsymbol{G} \boldsymbol{S}^{-1} \boldsymbol{C}_{f s} & \boldsymbol{H}-i k \beta \boldsymbol{G}
\end{array}\right]\left\{\begin{array}{l}
\boldsymbol{u} \\
\boldsymbol{p}
\end{array}\right\}=\boldsymbol{B}\left\{\begin{array}{l}
\boldsymbol{u} \\
\boldsymbol{p}
\end{array}\right\}=\left\{\begin{array}{l}
\boldsymbol{f}_{s} \\
\boldsymbol{p}_{i}
\end{array}\right\} .
$$

By differentiating equation (16) with respect to the design variable $\vartheta$, we can obtain the following formulation:

$$
\boldsymbol{B}\left\{\begin{array}{c}
\dot{\boldsymbol{u}} \\
\dot{\boldsymbol{p}}
\end{array}\right\}=\left\{\begin{array}{c}
\boldsymbol{r}_{1} \\
\boldsymbol{r}_{2}
\end{array}\right\}=\boldsymbol{r}
$$

in which

$$
\boldsymbol{r}=\left\{\begin{array}{c}
\dot{\boldsymbol{f}}_{s} \\
\dot{\boldsymbol{p}}_{i}
\end{array}\right\}-\dot{\boldsymbol{B}}\left\{\begin{array}{c}
\boldsymbol{u} \\
\boldsymbol{p}
\end{array}\right\}
$$

For different design variables, different expressions of $\dot{\boldsymbol{B}}$ could be obtained. When the fluid density $\rho_{f}$ is chosen as the design variable, $\dot{\boldsymbol{B}}$ could be expressed as:

$$
\dot{\boldsymbol{B}}=\left[\begin{array}{cc}
\boldsymbol{0} & \boldsymbol{0} \\
-\omega^{2} \boldsymbol{G} \boldsymbol{S}^{-1} \boldsymbol{C}_{f s} & \boldsymbol{0}
\end{array}\right],
$$

and the vector $\boldsymbol{r}$ could be expressed as:

$$
\boldsymbol{r}=\left\{\begin{array}{l}
\boldsymbol{r}_{1} \\
\boldsymbol{r}_{2}
\end{array}\right\}=\left\{\begin{array}{c}
\boldsymbol{0} \\
\omega^{2} \boldsymbol{G} \boldsymbol{S}^{-1} \boldsymbol{C}_{f s} \boldsymbol{u}
\end{array}\right\} .
$$

When the structural parameter is chosen as the design variable, such as the structural density $\rho_{s}$, Poisson's ratio $v$, Young's modulus $E$ or the thickness of the shell $h$ presented in the numerical example, $\dot{\boldsymbol{B}}$ could be expressed as: 


$$
\dot{B}=\left[\begin{array}{ll}
\dot{A} & 0 \\
0 & 0
\end{array}\right],
$$

and the vector $\boldsymbol{r}$ could be expressed as:

$$
\boldsymbol{r}=\left\{\begin{array}{c}
\boldsymbol{r}_{1} \\
\boldsymbol{r}_{2}
\end{array}\right\}=\left\{\begin{array}{c}
-\dot{\boldsymbol{A}} \boldsymbol{u} \\
\boldsymbol{0}
\end{array}\right\} .
$$

When the parameter which determines the structural nodal coordinates is chosen to be the design variable, such as the radius of the spherical shell in the numerical example, $\dot{\boldsymbol{B}}$ could be expressed as:

$$
\dot{\boldsymbol{B}}=\left[\begin{array}{cc}
\dot{\boldsymbol{A}} & -\dot{\boldsymbol{C}}_{s f} \\
-\omega^{2} \rho_{f}\left(\boldsymbol{G} \boldsymbol{S}^{-1} \boldsymbol{C}_{f s}\right) & \dot{\boldsymbol{H}}-i k \beta \dot{\boldsymbol{G}}
\end{array}\right],
$$

in which the upper dot $(\dot{)})$ denotes the differentiation with respect to the design variable. The vector $\boldsymbol{r}$ could be expressed as:

$$
\boldsymbol{r}=\left\{\begin{array}{c}
\boldsymbol{r}_{1} \\
\boldsymbol{r}_{2}
\end{array}\right\}=\left\{\begin{array}{c}
\dot{\boldsymbol{f}}_{s}-\dot{\boldsymbol{A}} \boldsymbol{u}+\dot{\boldsymbol{C}}_{s f} \boldsymbol{p} \\
\dot{\boldsymbol{p}}_{i}+\omega^{2} \rho_{f}\left(\boldsymbol{G} \boldsymbol{S}^{-1} \boldsymbol{C}_{f s}\right) \boldsymbol{u}-(\dot{\boldsymbol{H}}-i k \beta \dot{\boldsymbol{G}}) \boldsymbol{p}
\end{array}\right\} .
$$

When the acoustic admittance $\beta$ is set as the design variable, $\dot{\boldsymbol{B}}$ could be expressed as:

$$
\dot{\boldsymbol{B}}=\left[\begin{array}{cc}
\boldsymbol{0} & \boldsymbol{0} \\
\boldsymbol{0} & -i k \boldsymbol{G}
\end{array}\right],
$$

and the vector $\boldsymbol{r}$ could be expressed as:

$$
\boldsymbol{r}=\left\{\begin{array}{l}
\boldsymbol{r}_{1} \\
\boldsymbol{r}_{2}
\end{array}\right\}=\left\{\begin{array}{c}
\boldsymbol{0} \\
i k \boldsymbol{G} \boldsymbol{p}
\end{array}\right\} .
$$

In the equations shown above, the differentiation of the matrices could be very complicated and difficult. However, the semi-analytical derivative method, through which variations of the coefficient matrices could be calculated using the finite difference method, can be used to solve this problem. For example, matrix $\dot{\boldsymbol{D}}$ could be calculated using a small perturbation $\tau$ when the design variable is denoted by $\alpha$, as follows:

$$
\dot{\boldsymbol{D}}=\frac{\boldsymbol{D}(\alpha+\tau)-\boldsymbol{D}(\alpha)}{\tau} .
$$

In this paper, we use the step size $\tau / \alpha=1 \mathrm{e}-3$. Directly solving equation (17) could be quite inefficient because the system matrix will be very large in realistic problems. Reformulating equation (17), we can obtain the following equations: 


$$
\begin{gathered}
\boldsymbol{r}_{1}=\boldsymbol{A} \dot{\boldsymbol{u}}-\boldsymbol{C}_{s f} \dot{\boldsymbol{p}}, \\
\boldsymbol{r}_{2}=-\omega^{2} \rho_{f} \boldsymbol{G} \boldsymbol{S}^{-1} \boldsymbol{C}_{f s} \dot{\boldsymbol{u}}+(\boldsymbol{H}-i k \beta \boldsymbol{G}) \dot{\boldsymbol{p}} .
\end{gathered}
$$

By substituting equation (28) into equation (29), the new formulation could be obtained, as follows:

$$
(\boldsymbol{H}-i k \beta \boldsymbol{G}) \dot{\boldsymbol{p}}-\boldsymbol{G} \boldsymbol{W} \boldsymbol{C}_{s f} \dot{\boldsymbol{p}}=\boldsymbol{G} \boldsymbol{W} \boldsymbol{r}_{1}+\boldsymbol{r}_{2} .
$$

By solving the above equation, the sensitivity of the nodal sound pressure on the surface could be obtained. We can solve the unknown vector $\dot{\boldsymbol{u}}$ by substituting the solution of the vector $\dot{\boldsymbol{p}}$ into equation (28) and solving it.

\section{An example of scattering from an elastic spherical shell}

In this example, we consider the acoustic scattering of a plane incident wave with a unit amplitude on a spherical shell centered at point $(0,0,0)$ with radius $r=1.0 \mathrm{~m}$, with the wave travelling along the positive $\mathbf{x}$ axis, as shown in figure 1 . For the spherical shell, the density is $7860 \mathrm{~kg} / \mathrm{m}^{3}$, Young's modulus is $210 \mathrm{GPa}$, Poisson's ratio is 0.3 , thickness is $0.01 \mathrm{~m}$. For the fluid, the density is $1000 \mathrm{~kg} / \mathrm{m}^{3}$, speed of sound is $1482 \mathrm{~m} / \mathrm{s}$.

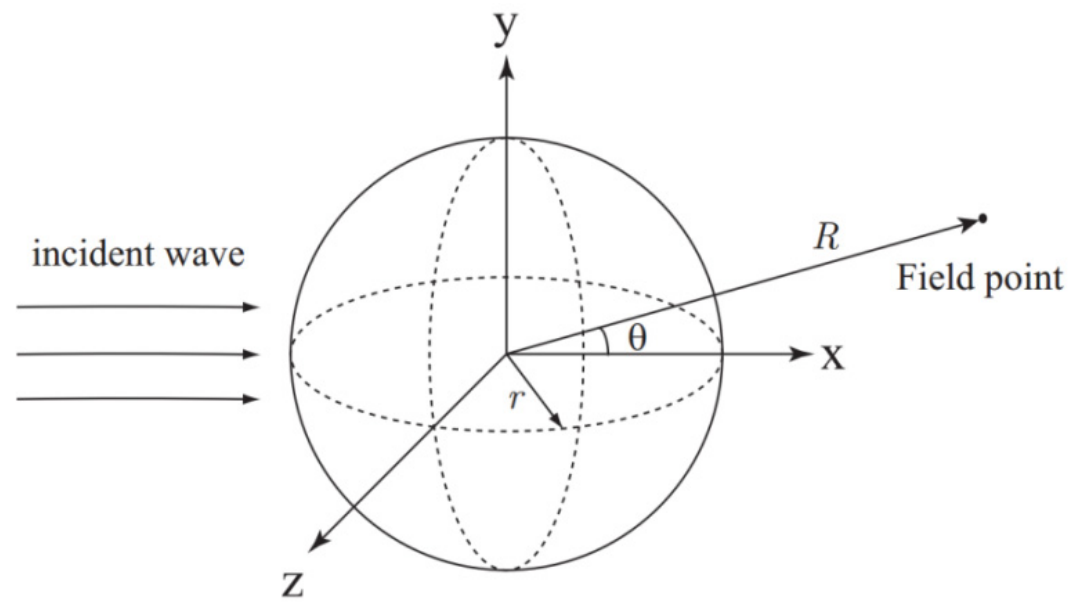

Figure 1: Scattering from a spherical shell with radius $r$.

Figures 2-4 give the analytical and the numerical solutions for the pressure sensitivity analysis at point $(2 r, 0,0)$, where the design variable is chosen to be the fluid density $\rho_{f}$, Young's modulus $E$ and radius $r$, respectively. These figures show that the scattered sound pressure remains rather insensitive in the low frequency range, and the sensitivity increases rapidly in the vicinity of resonance peaks. The numerical solution is in agreement with the analytical solution, which indicates the validity of the proposed algorithm and its correct implementation. 


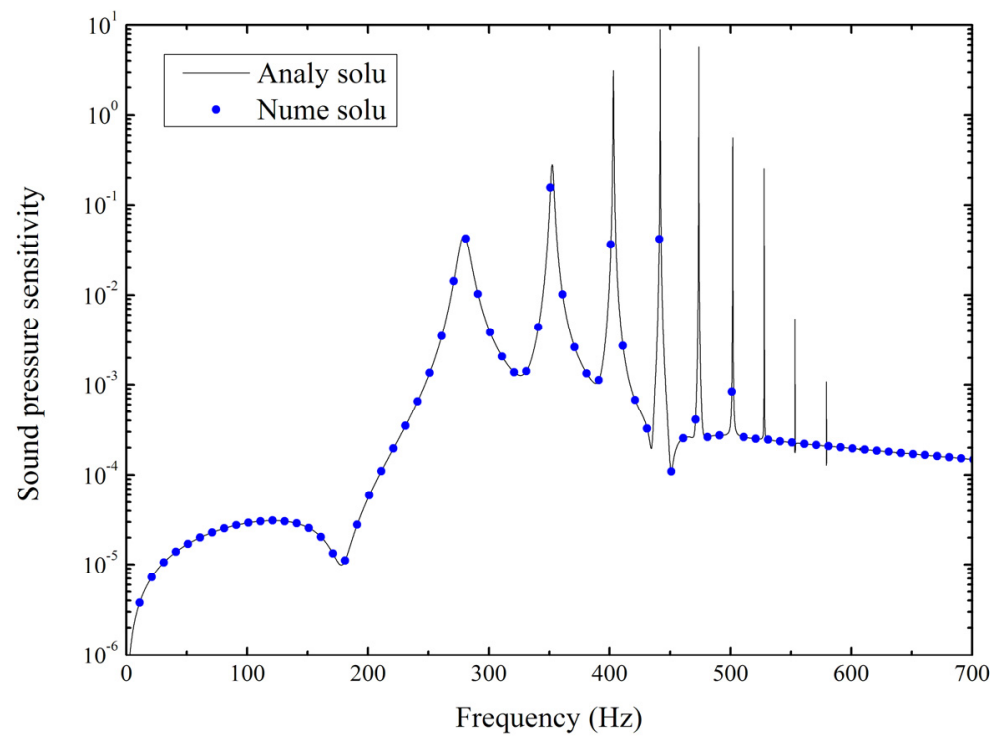

Figure 2: Sound pressure sensitivity with respect to fluid density $\rho f$.

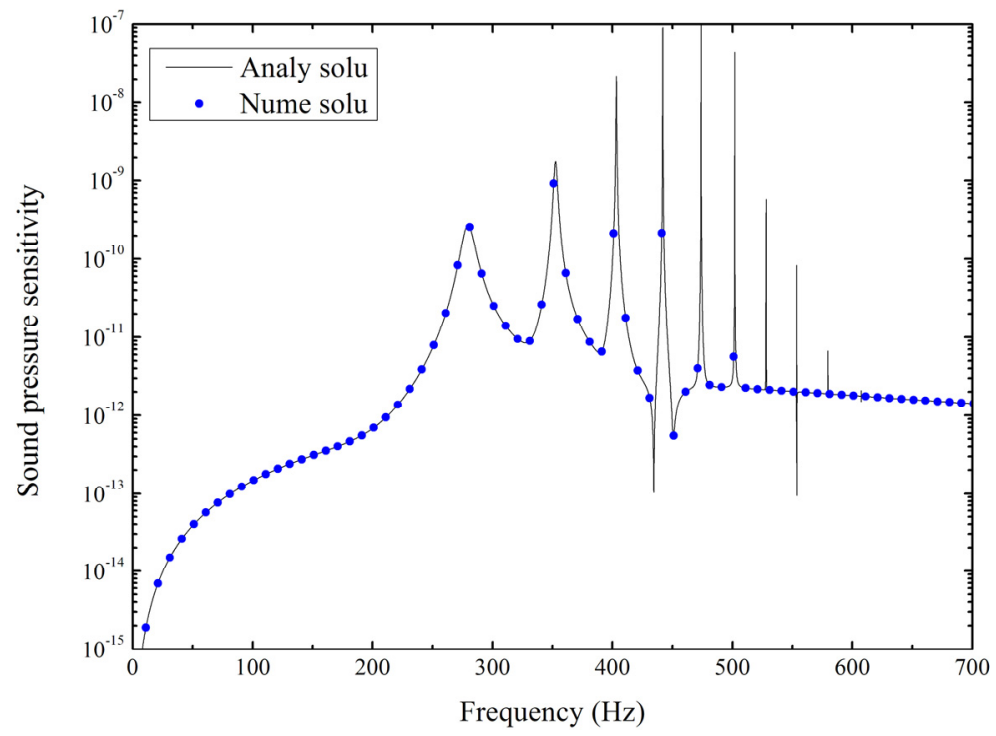

Figure 3: Sound pressure sensitivity with respect to Young's modulus E. 


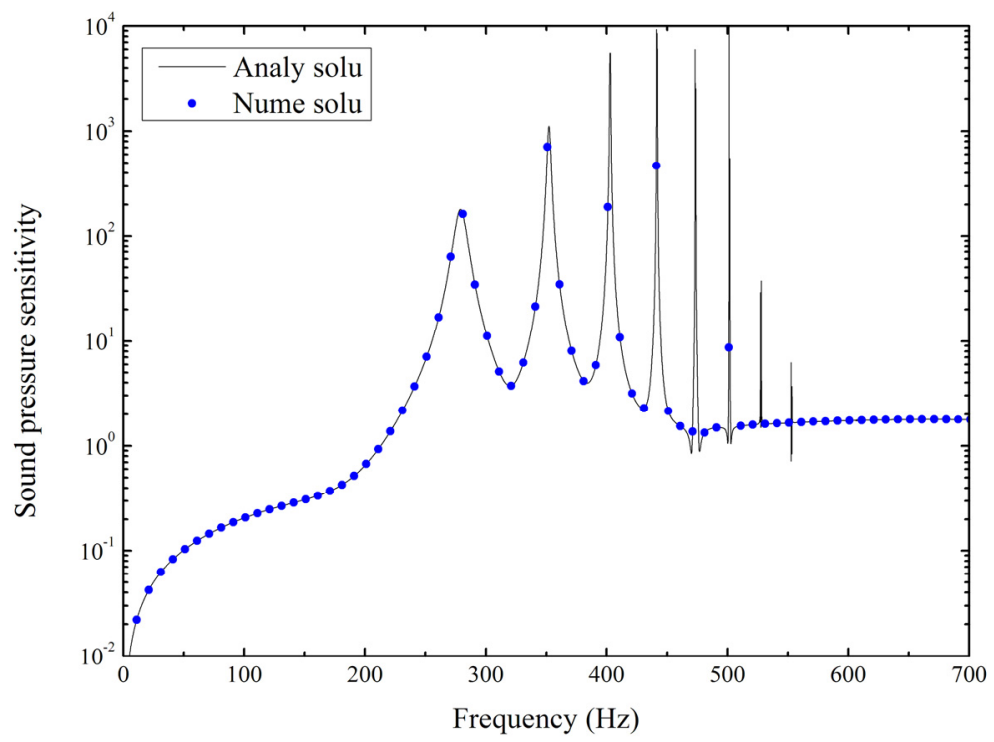

Figure 4: $\quad$ Sound pressure sensitivity with respect to radius $r$.

Figure 5 shows the scattered pressure sensitivity with respect to the acoustic admittance $\beta$ at $50 \mathrm{~Hz}$, where the computing points are located in a circle centered at point $(0,0,0)$ with radius $2 r$. The figure shows that the scattered sound pressure sensitivity varies slightly at different points.

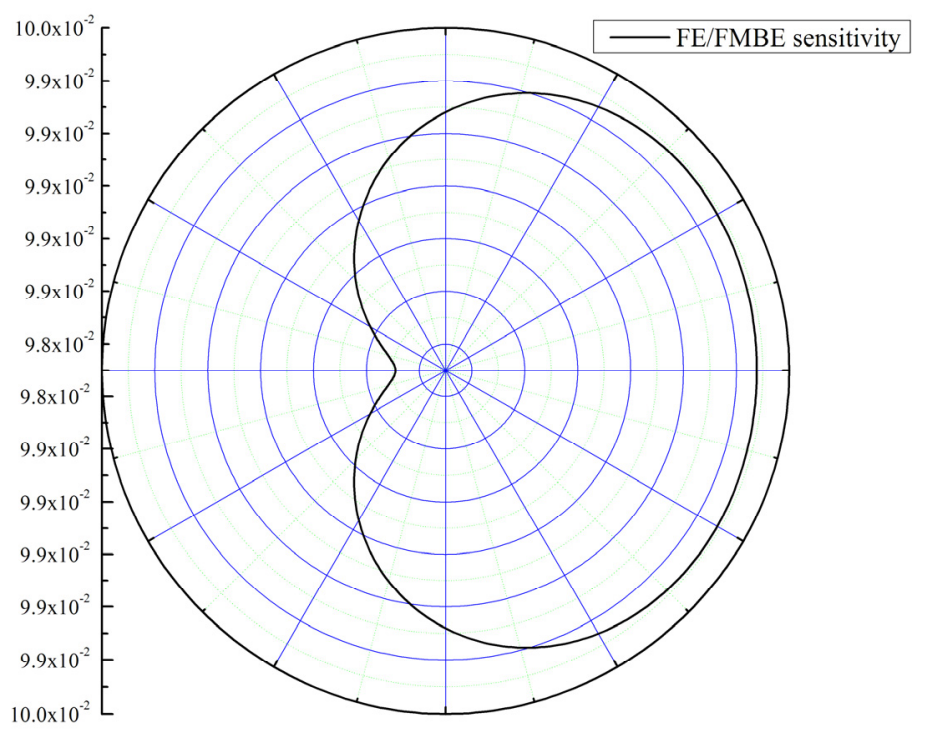

Figure 5: Sound pressure sensitivity with respect to acoustic admittance $\beta$. 
The CPU time used to calculate the acoustic pressure at point $(2 r, 0,0)$ is given in figure 6, in which BEM and FMBEM denote the solutions calculated by CBEM and FMBEM algorithm when interaction between fluid and structure is not considered (rigid analysis). We can see from the figure that elastic analysis requires much more time than rigid analysis. It can also be seen that the FEM/FMBEM algorithm shows high efficiency for three dimensional fluidstructure interaction analysis and sensitivity analysis.

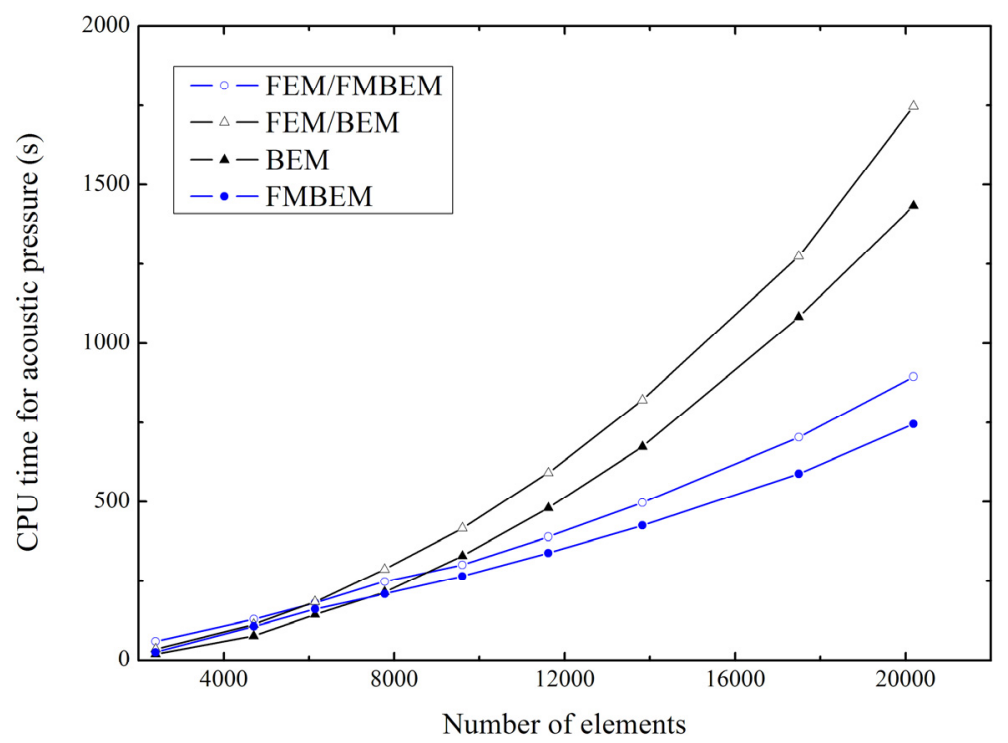

Figure 6: $\quad$ PUU time consumed to calculate the sound pressure with $\mathrm{f}=50 \mathrm{~Hz}$ at point $(2 r, 0,0)$.

\section{Conclusions}

A coupling algorithm based on FEM and BEM is presented for the simulation of fluid-structure interaction and structural acoustic sensitivity analysis using the direct differentiation method, in which several parameters are chosen as the design variable, e.g. Young's modulus, structural thickness, the acoustic admittance. For different design variables, the different formulations are presented for the calculation of the derivatives to the sound pressure. A numerical example is presented to demonstrate the validity of the proposed algorithm. The algorithm presented in this paper makes it possible to predict the effects of different design variables on the scattered sound field numerically. Future work will include applying the structural-acoustic design sensitivity analysis to optimize and extending the method to practical engineering problems. 


\section{Acknowledgements}

Financial support from the National Natural Science Foundation of China (NSFC) under Grant No. 11172291 and the Research Fund for the Doctoral Program of Higher Education of China under Grant No. 20133402110036 is acknowledged.

\section{References}

[1] Junger, M.C. \& Feit, D., Sound, structures, and their interaction, MIT Press, 1986.

[2] Everstine, G.C. \& Henderson, F.M., Coupled finite element/boundary element approach for fluid-structure interaction. Journal of the Acoustical Society of America, 87(5), pp. 1938-1947, 1990.

[3] Fritze, D., Marburg, S. \& Hardtke, H.J., FEM-BEM-coupling and structural-acoustic sensitivity analysis for shell geometries. Computers \& Structures, 83(2-3), pp. 143-154, 2005.

[4] Greengard, L. \& Rokhlin, V., A fast algorithm for particle simulations. Journal of Computational Physics, 73(2), pp. 325-348, 1987.

[5] Coifman, R., Rokhlin, V. \& Wandzura, S., The fast multipole method for the wave equation: A pedestrian prescription. IEEE Antennas and Propagation Magazine, 35(3), pp. 7-12, 1993.

[6] Song, J.M., Lu, C.C. \& Chew, W.C., Multilevel fast multipole algorithm for electromagnetic scattering by large complex objects. IEEE Transactions on Antennas and Propagation, 45(10), pp. 1488-1493, 1997.

[7] Shen, L. \& Liu, Y.J., An adaptive fast multipole boundary element method for three-dimensional acoustic wave problems based on the Burton-Miller formulation. Computational Mechanics, 40(3), pp. 461-472, 2007.

[8] Zheng, C.J., Chen, H.B., Matsumoto, T. \& Takahashi, T., 3D acoustic shape sensitivity analysis using fast multipole boundary element method. International Journal of Computational Methods, 9(1), pp. 1240004-11240004-11, 2012.

[9] Cheng, H.W., Crutchfield, W.Y., Gimbutas, Z., Greengard, L.F., Ethridge, J.F., Huang, J.F., Rokhlin, V., Yarvin, N. \& Zhao, J.S., A wideband fast multipole method for the Helmholtz equation in three dimensions. Journal of Computational Physics, 216(1), pp. 300-325, 2006.

[10] Gumerov, N.A. \& Duraiswami, R., A broadband fast multipole accelerated boundary element method for the three dimensional Helmholtz equation. Journal of the Acoustical Society of America, 125(1), pp. 191-205, 2009.

[11] Zheng, C.J., Matsumoto, T., Takahashi, T. \& Chen, H.B., A wideband fast multipole boundary element method for three dimensional acoustic shape sensitivity analysis based on direct differentiation method. Engineering Analysis with Boundary Elements, 36(3), pp. 361-371, 2012.

[12] Burton, A.J. \& Miller, G.F., The application of integral equation methods to the numerical solution of some exterior boundary-value problems. Proceedings of the Royal Society of London Series A - Mathematical and Physical Sciences, 323(1553), pp. 201-210, 1971. 
[13] Chen, L.L., Zheng, C.J. \& Chen, H.B., FEM/wideband FMBEM coupling for structural-acoustic design sensitivity analysis. Computer Methods in Applied Mechanics and Engineering, 276, pp. 1-19, 2014.

[14] Lamarque, N. \& Poinsot, T., Boundary conditions for acoustic eigenmode computations in gas turbine combustion chambers. AIAA Journal, 46(9), pp. 2282-2292, 2008.

[15] Kim, S.K., Choi, H.S., Kim, H.J., Ko, Y.S. \& Sohn, C.H., Finite element analysis for acoustic characteristics of combustion stabilization devices. Aerospace Science and Technology, 42, pp. 229-240, 2015.

[16] Chen, L.L., Zheng, C.J. \& Chen, H.B., A wideband FMBEM for 2D acoustic design sensitivity analysis based on direct differentiation method. Computational Mechanics, 52(3), pp. 631-648, 2013. 